\title{
Metodología para el cálculo de espesores límite libres de fase sigma durante el hipertemple en piezas de aceros dúplex de gran sección ${ }^{(\cdot)}$
}

\author{
P. Jimbert ${ }^{*}$, T. Guraya*, A. Torregaray* y P. Bravo**
}

\begin{abstract}
Resumen
Para conseguir las propiedades mecánicas y de corrosión deseadas en los aceros dúplex utilizados por la industria petro-química y nuclear se les somete a un tratamiento de hipertemple desde unos $1.050^{\circ} \mathrm{C}$. Con ello se evita el riesgo de aparición de precipitados intermetálicos que reducen drásticamente las propiedades de estos materiales. Sin embargo, al aumentar la profundidad a la que se encuentran los yacimientos actuales, los espesores para este tipo de canalizaciones se han visto incrementados, lo que se traduce en mayores niveles de exigencia sobre todo su proceso de fabricación, incluyendo el tratamiento térmico ${ }^{[1]}$. Para evitar la precipitación de estas fases intermetálicas, como la fase sigma, es necesario conocer el perfil de enfriamiento en el centro de la pieza y para ello, conocer el valor del Coeficiente de Transferencia de Calor Superficial (h) es fundamental. Dicho coeficiente varía durante el hipertemple y su valor se determina experimentalmente ya que depende de varios parámetros del proceso. Diversos estudios demuestran que su valor se estabiliza a los pocos segundos, por lo que para conocer el perfil de enfriamiento en el centro de grandes secciones bastaría con conocer el valor estabilizado de $h$. Sin embargo, los estudios existentes en la literatura se refieren a diámetros menores de $100 \mathrm{~mm}$. En el presente trabajo se ha desarrollado una metodología para predecir la precipitación de fases intermetálicas en piezas de acero dúplex con grandes espesores en instalaciones industriales a partir del cálculo de h. Esta metodología nos permite calcular los perfiles de enfriamiento sin sacrificar ninguna pieza, utilizando una o varias piezas patrón sensorizadas con termopares y una simulación posterior mediante ANSYS.
\end{abstract}

Palabras clave Fase sigma; Acero dúplex; Coeficiente de transferencia de calor superficial.

\section{Methodology for calculating the thickness free of sigma phase in duplex stainless steels large section parts during hiperquenching}

\begin{abstract}
To achieve the mechanical properties and corrosion resistance desired by duplex stainless steels used by the petrochemical and nuclear industry, parts are subjected to a hiperquenching heat treatment from about $1050^{\circ} \mathrm{C}$. This avoids the risk of intermetallic precipitation which drastically reduces the properties of these materials. However with increasing depth to which the deposits are present, the thicknesses for such pipes have been increased, resulting in higher levels of demand on all its manufacturing process, including the heat treatment. To avoid the precipitation of intermetallic phases such as sigma phase it is necessary to know the cooling profile in the center of the workpiece and for this purpose to know the value of the Surface Heat Transfer Coefficient $(h)$ is essential. This coefficient changes during the hiperquenching and its value is determined experimentally as it depends on several process parameters. Studies reveal that its value is stabilized within a few seconds. We can then assume that to know the cooling profile in the center of large sections it is only necessary to know the stabilized value of h. However, all the studies found in the literature are referred to diameters smaller than $100 \mathrm{~mm}$. This paper has developed a methodology to predict the precipitation of intermetallic phases in duplex stainless steel parts with large thicknesses in industrial facilities from the calculation of $\mathrm{h}$. This methodology allows us to calculate the cooling profiles without wasting any workpiece using one or more sensorized patterns with thermocouples and a subsequent simulation with ANSYS.
\end{abstract}

Keywords

Sigma-phase; Duplex stainless steel; Surface heat transfer coefficient.

(•) Trabajo recibido el día 25 de julio de 2012 y aceptado en su forma final el día 19 de noviembre de 2012.

EUITI, Universidad del País Vasco UPV/EHU, PI. La Casilla 3, 48012, Bilbao, Spain.

T. Gainza, S.L., B. Agote 7, 20740, Zestoa, Spain. 


\section{INTRODUCCIÓN}

El uso de aceros dúplex y superdúplex para la construcción de canalizaciones submarinas de combustibles está en aumento a nivel mundial ${ }^{[2]}$ debido a sus excelentes características mecánicas combinadas con una gran resistencia a la corrosión y a la corrosión bajo tensión. La figura 1 permite comparar el consumo de los aceros dúplex con el de otros inoxidables y el de aleaciones base níquel.

Sin embargo, el progresivo agotamiento de los yacimientos más próximos a la plataforma continental está llevando a que las explotaciones tengan lugar en aguas cada vez más profundas. Las tuberías que transporten el combustible desde estos lugares de extracción más remotos tienen que operar bajo presiones mucho mayores que las actuales, lo que exige conductos de mayores espesores para poder soportar esas presiones. Además, el reciente vertido sucedido en una plataforma de extracción en el golfo de México y la catástrofe ocurrida en Fukushima han hecho que el nivel de calidad exigido a estas piezas sea cada vez mayor. Todo ello exige un mayor conocimiento sobre el procesado de estos materiales para asegurar su perfecto rendimiento en los nuevos espesores demandados por la industria.

Uno de los aspectos más importantes a tener en cuenta con estos materiales es que los aceros dúplex tienen una cantidad de elementos de aleación que los hacen susceptibles de precipitar fases intermetálicas durante su procesamiento. En general son fases frágiles, ricas en cromo y molibdeno que generan a su alrededor una zona empobrecida en esos elementos, afectando a su resistencia a la corrosión y a las propiedades mecánicas. Para evitar esta precipitación las piezas sufren un tratamiento térmico de solubilización de esas fases frágiles a unos $1.050^{\circ} \mathrm{C}$ y posteriormente se realiza un hipertemple. Sin embargo, al aumentar el espesor de este tipo de piezas surge la duda de si la nueva velocidad de enfriamiento generada en el centro de la pieza durante el hipertemple es lo suficientemente alta para evitar la precipitación de fases intermetálicas. A nivel nacional son numerosos los estudios que tratan el problema de la precipitación de fases intermetálicas en los aceros dúplex. Existen estudios sobre la cinemática de las transformaciones de estas fases intermetálicas ${ }^{[3]}$, sobre su influencia en las propiedades mecánicas y en servicio ${ }^{[4]}$, sobre la influencia en su conformabilidad ${ }^{[5]}$ o sobre los problemas que surgen al ser soldados ${ }^{[6]}$. Sin embargo, todos los estudios encontrados se refieren a ensayos a escala de laboratorio y no existen referencias sobre procesos industriales con piezas de gran tamaño.

Hoy en día existen programas de simulación capaces de predecir perfiles de enfriamiento en cualquier geometría, sin embargo es necesario conocer algunos datos a introducir. Uno de los parámetros que más influyen en la velocidad de enfriamiento es el coeficiente de transferencia de calor superficial, $h$ $\left(\mathrm{W} / \mathrm{m}^{2} \mathrm{~K}\right)$. Este coeficiente además de ser fundamental para calcular el perfil de enfriamiento y las características mecánicas finales de las piezas durante el temple, depende de las características propias del baño (propiedades del fluido, naturaleza del movimiento si es forzado, su velocidad masiva) y de las constantes térmicas del material y su configuración geométrica.

El valor de $\mathrm{h}$ cambia en las distintas etapas del temple en función de la temperatura superficial de la pieza ${ }^{[7]}$. Su valor, en primer lugar, es bajo debido a una pantalla

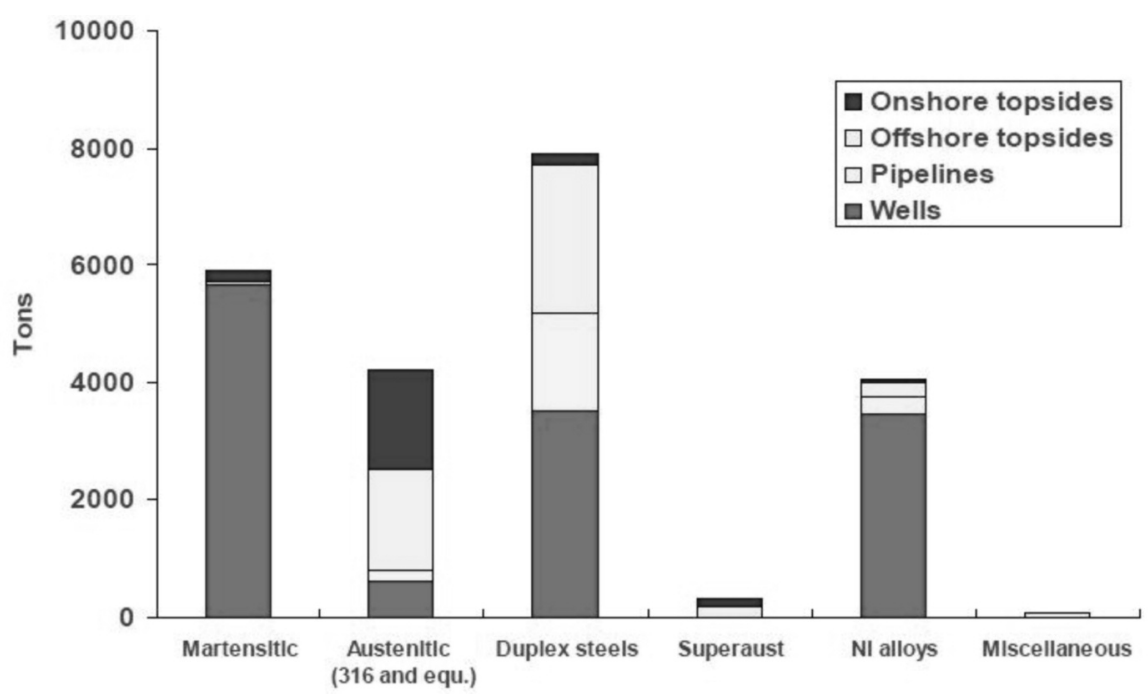

Figura 1. Consumo de aleaciones resistentes a la corrosión en diferentes proyectos en el mundo[2].

Figure 1. Corrosion resistant alloys consumption worldwide ${ }^{[2]}$. 
de gas que cubre la pieza y que aísla la misma, lo cual se traduce en una velocidad de enfriamiento baja. A continuación esa capa se va destruyendo progresivamente y pasa a un estado de ebullición en la superficie que aumenta la velocidad de enfriamiento debido al movimiento del agua. Finalmente esta ebullición disminuye, y con ella la agitación, por lo que $h$ desciende progresivamente hasta que desaparece la ebullición y dicho parámetro h se estabiliza en un valor final ${ }^{[8]}$ (Fig. 2).

Utilizando métodos numéricos y programas de simulación se ha conseguido calcular su valor para las distintas etapas del temple por el método inverso de transferencia de calor, obteniendo valores de la evolución de h precisos en el tiempo para cada caso estudiado $^{[9-13]}$. Este método ha sido muy utilizado debido a su aplicabilidad en procesos tecnológicos ya que solamente es necesario conocer la temperatura en ciertos puntos interiores de la pieza para poder calcular $h$.

En los últimos años se han realizado diversos estudios al respecto ${ }^{[14 \text { y } 15]}$. L. Huiping et al. ${ }^{[16]}$ han comparado los valores de $\mathrm{h}$ obtenidos para experimentos con parámetros similares, sin embargo los resultados obtenidos no son homogéneos, lo que delata la complejidad de dicho cálculo (Fig. 3). Además, los estu-

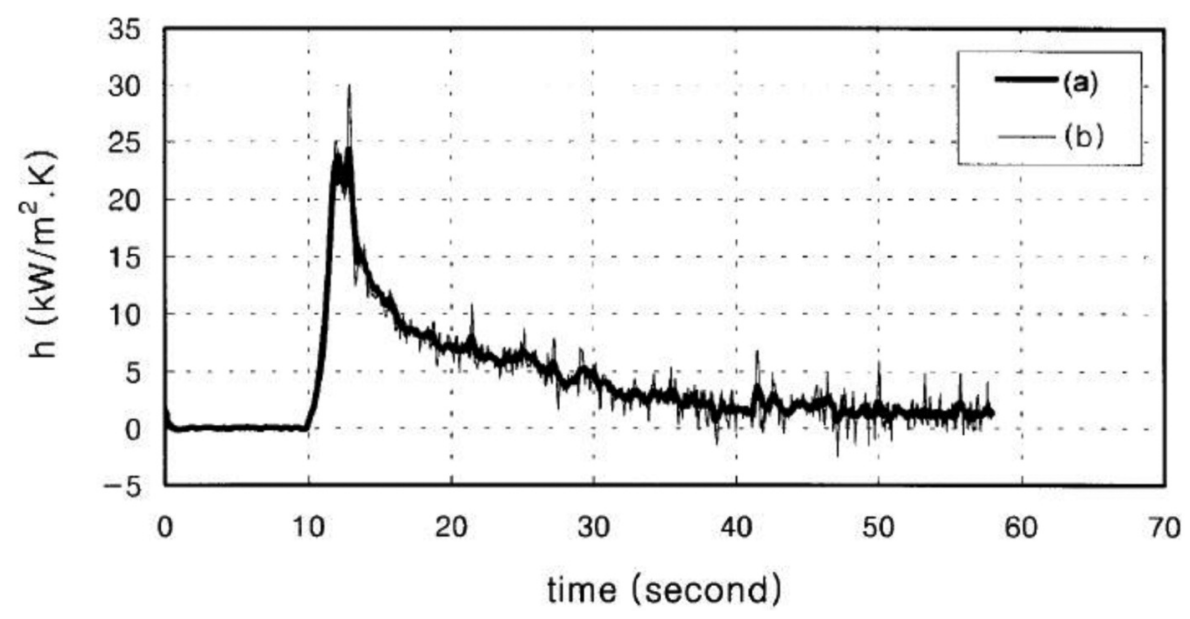

Figura 2. Evolución de h durante el temple ${ }^{[8]}$.

Figure 2. $h$ evolution during quenching ${ }^{[8]}$.

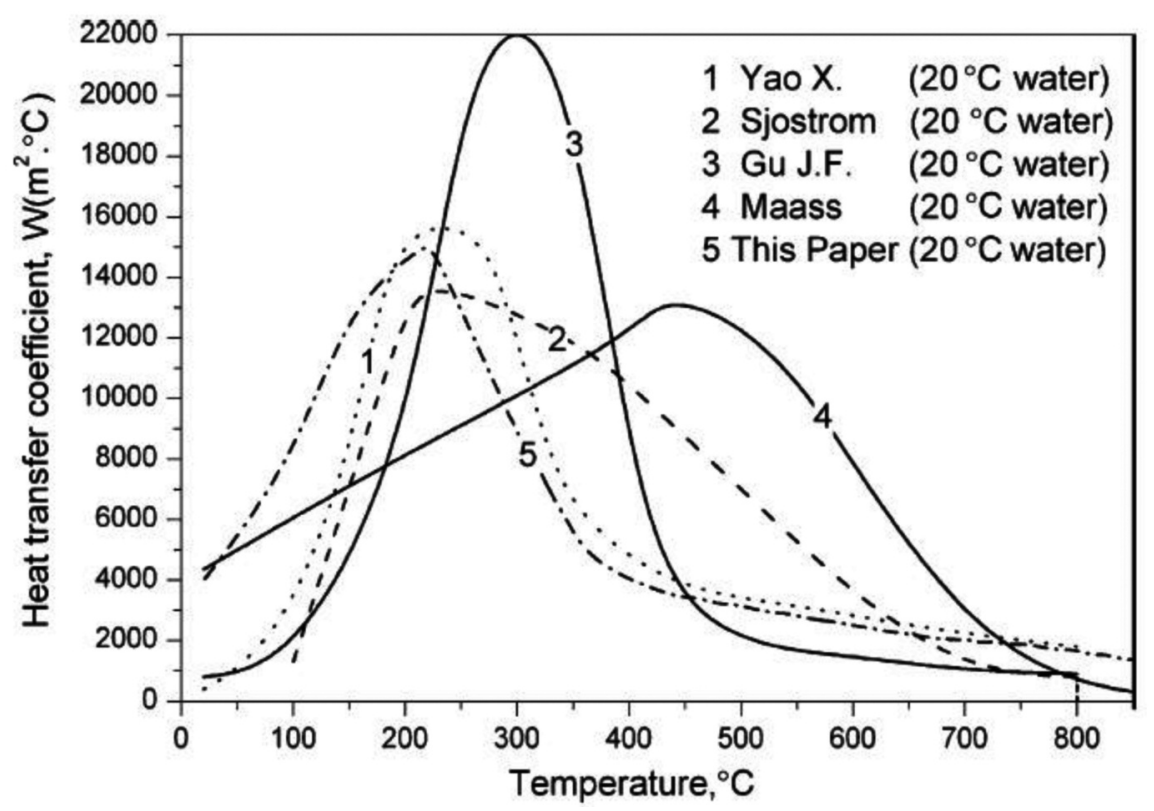

Figura 3. Valores de h obtenidos por distintos investigadores para condiciones similares ${ }^{[16]}$.

Figure 3. Different $h$ values obtained by different researchers for similar testing conditions ${ }^{[16]}$. 
dios sobre el cálculo del valor de h encontrados y referenciados en este trabajo se refieren a piezas de no más de $100 \mathrm{~mm}$ de diámetro y a tiempos de temple inferiores al minuto, por lo que no son aplicables a piezas de secciones gruesas.

Un estudio más reciente de C. Heming et al. ${ }^{[17]}$ viene a demostrar esto último, es decir, que el valor de h obtenido durante todo el temple no es el mismo para piezas de distintos diámetros (Fig. 4).

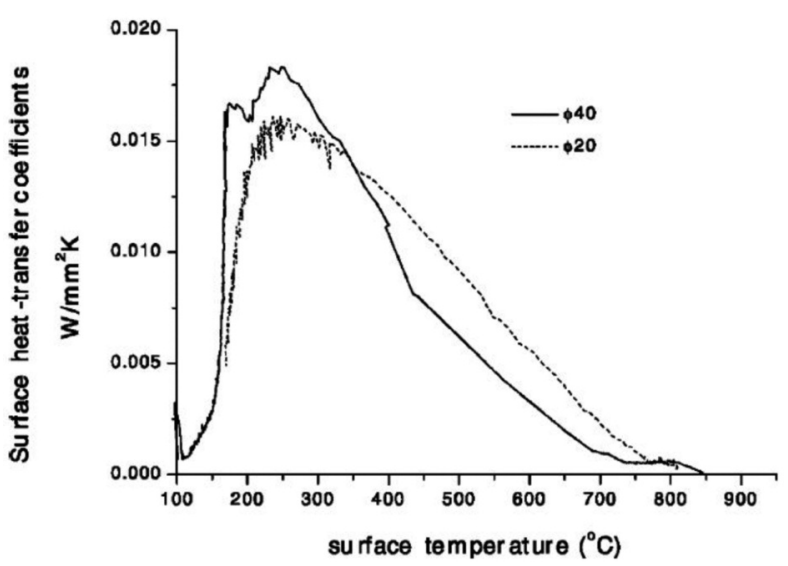

Figura 4. Valores de h para distintos diámetros de 20 y $40 \mathrm{~mm}^{[17]}$.

Figure 4. $h$ values for different diameters of 20 and $40 \mathrm{~mm}^{[17]}$.

Sin embargo, y a pesar de las desviaciones obtenidas en el cálculo sobre la evolución de h durante el temple, en la mayoría de los casos y para piezas de secciones pequeñas (menos de $100 \mathrm{~mm}$ ) su valor se estabiliza pasados unos segundos entre $800 \mathrm{y}$ $2000 \mathrm{~W} / \mathrm{m}^{2} \mathrm{~K}$ [14, 15, 18 y 19]. Se ha detectado, por lo tanto, una falta de estudios encaminados a conocer el valor de h para un caso industrial con piezas de grandes secciones y con parámetros de temple reales.

Para este estudio, y basándonos en lo encontrado en la bibliografía al respecto, se ha realizado la siguiente hipótesis: para calcular el perfil de enfriamiento en el interior de piezas de gran sección no es preciso calcular el valor de h en cada instante del tem- ple, sino el de la zona estabilizada, ya que lo importante es conocer el perfil de enfriamiento del interior de la pieza, que para espesores de $300 \mathrm{~mm}$ puede tardar cerca de media hora, mientras que h se estabiliza a los pocos segundos (Fig. 2). Esto último se comprobará en las simulaciones realizadas, calculando el tiempo que tarda la superficie de la pieza en alcanzar los $100^{\circ} \mathrm{C}$, temperatura de estabilización de $\mathrm{h}$.

En el presente estudio se han medido temperaturas durante el hipertemple en el interior de una pieza cilíndrica patrón de $300 \mathrm{~mm}$ de diámetro y también en una pieza anular real de sección variable con un espesor máximo de $170 \mathrm{~mm}$, que se ha denominado conector. Posteriormente se han calculado los valores de h estabilizada que mejor se ajustan a los perfiles obtenidos experimentalmente durante el enfriamiento utilizando el programa de elementos finitos ANSYS para la pieza cilíndrica y se han comprobado los resultados obtenidos para el conector. Una vez calculados los valores de h para el caso estudiado (tamaño del baño, temperatura del agua, tipo de agitación,...) y mediante el mismo programa de simulación, se podrá calcular el espesor máximo templable evitando el riesgo de precipitación de fase sigma comparando los resultados obtenidos con las curvas TTT del material. Este mismo procedimiento ha sido estudiado por Hasan et al. ${ }^{[20]}$ con buenos resultados pero aplicado nuevamente a una pieza pequeña de $52 \mathrm{~mm}$ de diámetro.

\section{PROCEDIMIENTO EXPERIMENTAL}

El material con el que se ha trabajado se suministró por la empresa Talleres Gainza S.L. y se trata de un acero inoxidable dúplex, con una composición similar a la que se muestra en la tabla I.

Para los ensayos físicos se instrumentalizaron la pieza cilíndrica de $300 \mathrm{~mm}$ de diámetro y el conector. Se colocaron termopares de tipo $\mathrm{k}$ a distintas profundidades para medir los distintos perfiles de enfriamiento generados, tal y como se puede ver en la figura 5 .

Los valores registrados por los termopares se derivaron hasta un sistema acondicionador de señales de

Tabla I. Composición química (\% en peso), del acero SAF 2205 estudiado

Table I. Chemical composition for SAF 2205 steel (weight \%)

\begin{tabular}{lcccccccccc}
\hline $\mathbf{C}$ & $\mathbf{M n}$ & $\mathbf{P}$ & $\mathbf{S}$ & $\mathbf{S i}$ & $\mathbf{C r}$ & $\mathbf{N i}$ & $\mathrm{Mo}$ & $\mathbf{N}$ & $\mathbf{C u}$ & $\mathbf{C o}$ \\
\hline 0,018 & 1,09 & 0,028 & 0,0004 & 0,53 & 22,35 & 5,35 & 3,12 & 0,181 & 0,19 & 0,045 \\
\hline
\end{tabular}


la marca Somat que las filtra y las convierte en digital. La velocidad de recogida fue de 1 dato por segundo. El tratamiento de hipertemple que se realizó fue el siguiente:

- Las piezas se calentaron durante aproximadamente $12 \mathrm{~h}$ hasta los $1.050^{\circ} \mathrm{C}$ para luego introducirlas en una piscina de agua que se encontraba a $16^{\circ} \mathrm{C}$. Dicho baño de agua consistía en una piscina de $170 \mathrm{~m}^{3}$ de volumen en la que se introdujo la hornada de $12.500 \mathrm{~kg}$ durante aproximadamente $30 \mathrm{~min}$. La piscina cuenta con un sistema que remueve el agua con toberas creando una agitación de $0,123 \mathrm{~m}^{3} / \mathrm{s}$.
De estos experimentos se obtuvieron las curvas de enfriamiento por los distintos termopares tanto para el cilindro como para el conector.

\section{RESULTADOS Y DISCUSIÓN}

Para calcular h se han estudiado los datos de perfiles de temperatura medidos en la pieza cilíndrica. Posteriormente los resultados se han aplicado al estudio del conector.
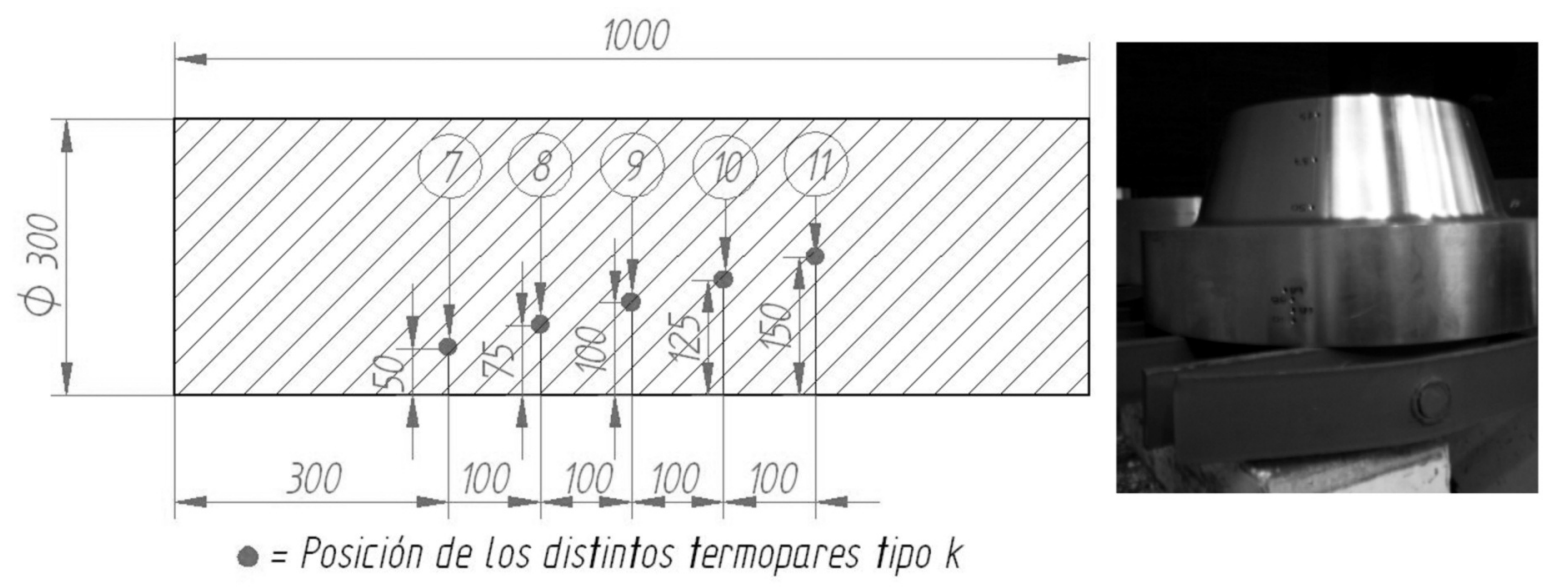

- Posición de los distintos termopares tipo k

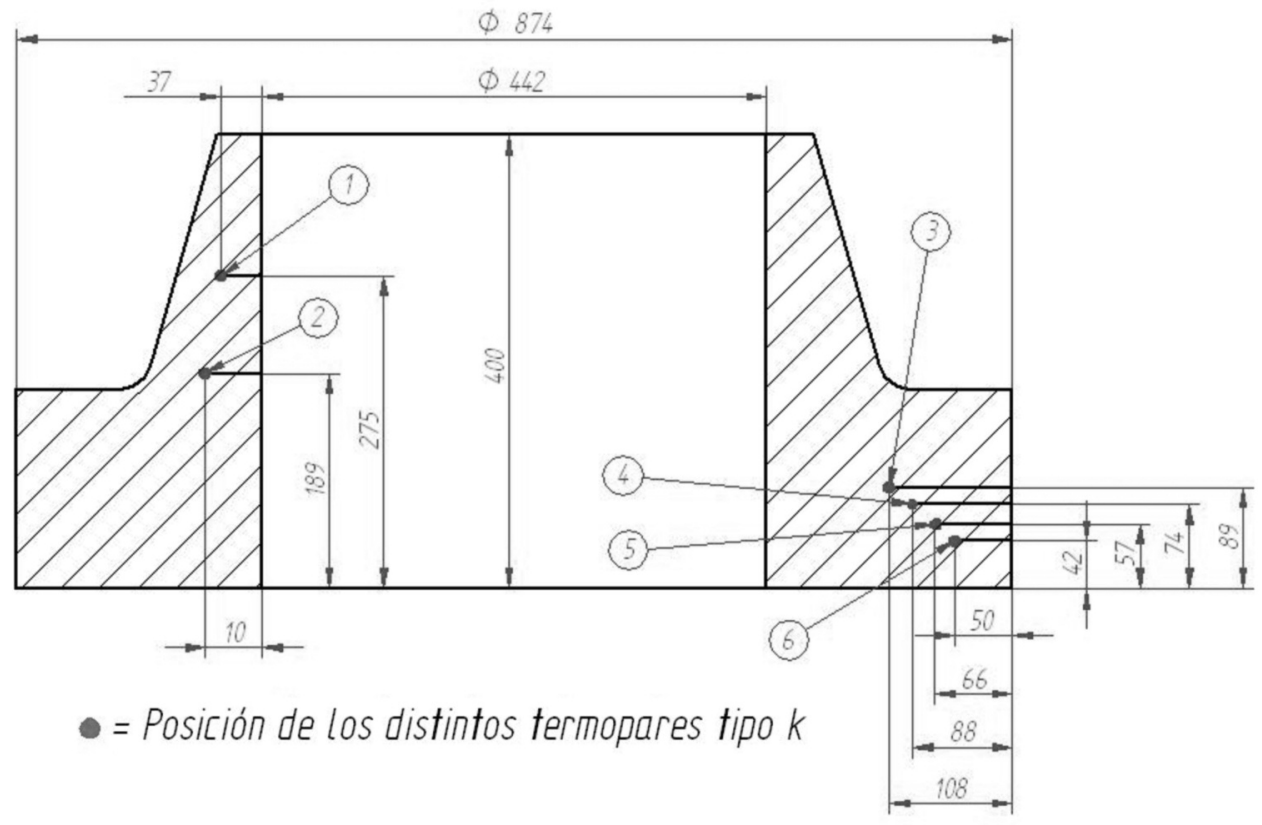

Figura 5. Profundidades de colocación de los termopares para las dos geometrías estudiadas (arriba izquierda: el cilindro y abajo: el conector) y foto del conector dentro del horno industrial (arriba a la derecha). Distancias en $\mathrm{mm}$.

Figure 5. Thermocuples positions for both geometries (top left: the cilinder and bottom: the connector) and picture of the connector inside the industrial oven (top right). Distances in $\mathrm{mm}$. 
En primer lugar, realizaremos una simulación de las condiciones del proceso del temple en la pieza cilíndrica, utilizada como pieza testigo en los ensayos. Posteriormente, estudiaremos el conector partiendo de las conclusiones a las que se llegue en el estudio del cilindro, ya que han sido sometidos al mismo tratamiento térmico en las mismas condiciones experimentales.

Utilizando ANSYS se modelizó la pieza cilíndrica. Se introdujeron los datos referentes al material estudiado además de los valores de la conductividad térmica $(\mathrm{k})$ y calor específico $\left(\mathrm{C}_{\mathrm{p}}\right)$ los cuales varían con la temperatura (Tabla II).

Para las simulaciones se realizaron las siguientes hipótesis de trabajo:

- Se trabajará con una sección axisimétrica del cilindro y del conector.

- Se trata de un proceso en régimen transitorio (la temperatura varía con el tiempo y la posición) donde se produce la variación del medio con el tiempo o dependencia con respecto a él.

- Al considerar el proceso en régimen transitorio se está admitiendo la suposición de sistema no concentrado, ya que la temperatura varía con el tiempo y no se puede considerar uniforme a través del sistema en todo momento.

- La agitación del agua la tendremos en cuenta desde el punto de vista de la convección y queda reflejada en el parámetro $h$.

- Se trata de un material isotrópico, es decir, que tiene propiedades uniformes en todas las direcciones.

- Las cargas que se aplicarán a las piezas, en nuestro caso, convección, se considera que se aplicarán axisimétricamente.

El elemento seleccionado para el análisis es el PLANE55, que puede ser utilizado como elemento axisimétrico para casos de conducción en 2D. El elemento posee cuatro nodos con la temperatura como único grado de libertad en ellos.

A continuación se introdujeron diferentes valores de h hasta conseguir ajustar la curva obtenida por simulación a la curva de enfriamiento experimental para el caso del cilindro. Al alcanzar $\mathrm{h}=3.100 \mathrm{~W} / \mathrm{m}^{2} \mathrm{~K}$ observamos el mayor ajuste entre las curvas experi- mentales y las obtenidas mediante simulación, logrando un solapamiento prácticamente perfecto en el centro de la pieza. En la figura 6 solamente se han representado el termopar menos profundo y el más profundo para facilitar su visualización.

Se puede observar que ese ajuste no es tan bueno en los primeros instantes del temple. En esos primeros instantes h varía (tal y como hemos comentado anteriormente) pero una vez pasado ese tiempo se estabiliza en un valor constante que una vez ajustado y calculado nos da el perfil en el interior de estas piezas de gran sección.

Una vez ajustado el valor de h estable para el tratamiento realizado, se simuló directamente el conector con ese mismo valor calculado de $3.100 \mathrm{~W} / \mathrm{m}^{2} \mathrm{~K}$ y se obtuvo el perfil de enfriamiento en el punto más alejado de cualquier superficie. En la figura 7 se puede observar cómo los resultados obtenidos por ANSYS se ajustan perfectamente a los experimentales, prediciendo el perfil de enfriamiento con gran exactitud en la zona de mayor riesgo de precipitados intermetálicos que va de los 950 a los $650^{\circ} \mathrm{C}$.

El mayor error producido entre ambas curvas está en torno al minuto para este proceso que dura media hora. De todos modos, al tratarse de geometrías diferentes es normal ese error. Introduciendo testigos con diferentes geometrías tipo se obtendrían resultados con mayor exactitud para las distintas piezas tratadas.

De estas mismas simulaciones se observa que, a pesar del gran tamaño de las piezas analizadas, la $\mathrm{h}$ variable debida a las etapas de capa de vapor y posterior ebullición dura pocos segundos ya que la temperatura superficial alcanza los $100-200^{\circ} \mathrm{C}$ en $50 \mathrm{~s}$ (Fig. 8). A partir de aquí h se estabiliza. Calcular este valor estable es por lo tanto suficiente para poder predecir el enfriamiento en el centro de la pieza.

Queda pues demostrado que el valor de h para predecir el enfriamiento en el centro de piezas de gran sección para el caso estudiado es de $3.100 \mathrm{~W} / \mathrm{m}^{2} \mathrm{~K}$. Una vez conocido este dato se puede calcular el espesor máximo templable en esta instalación libre de fases frágiles tales como sigma. Para

Tabla II. Valores de conductividad térmica $(k)$ y calor específico $\left(C_{p}\right)$ del acero SAF 2205 estudiado

Table II. Thermal conductivity $(k)$ and specific heat $\left(C_{p}\right)$ for the SAF 2205 alloy

\begin{tabular}{lcccccrr}
\hline Temperatura $\left({ }^{\circ} \mathrm{C}\right)$ & 27 & 127 & 327 & 527 & 727 & 927 & 1.127 \\
$\mathrm{k}(\mathrm{W} / \mathrm{mK})$ & 13,4 & 15,2 & 18,3 & 21,3 & 24,2 & 28 & 32 \\
$\mathrm{C}_{\mathrm{p}}(\mathrm{J} / \mathrm{kgK})$ & 468 & 504 & 550 & 576 & 602 & 610 & 620 \\
\hline
\end{tabular}




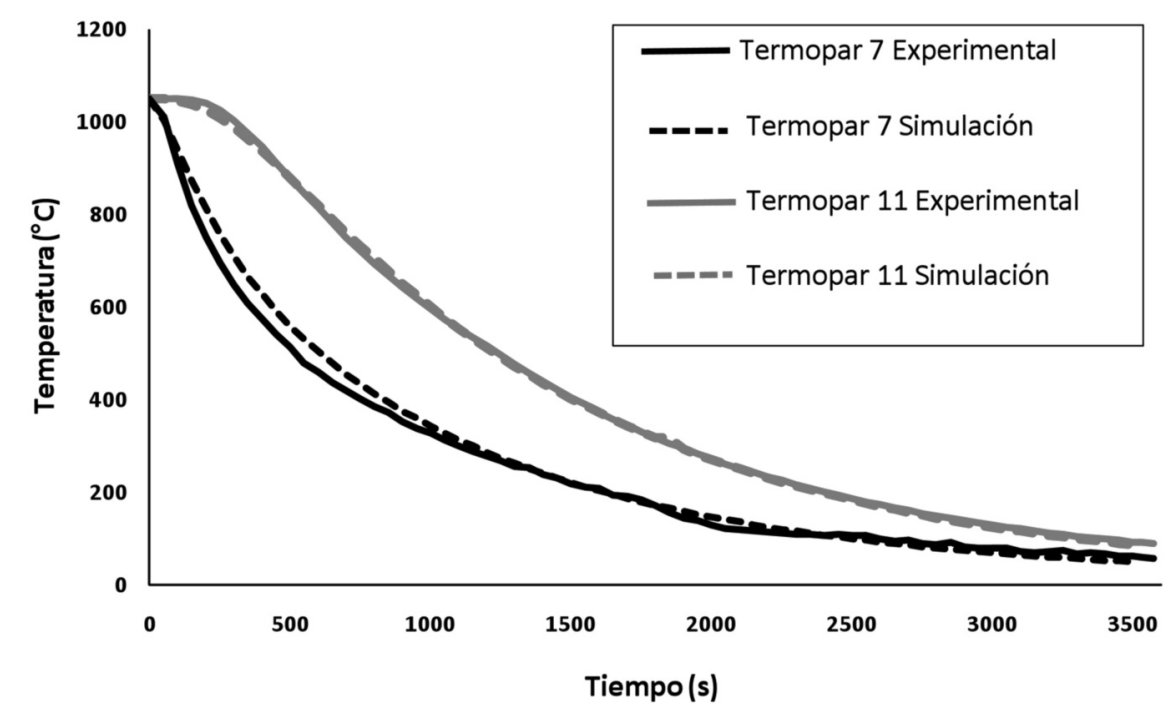

Figura 6. Perfiles de enfriamiento obtenidos en el cilindro por los termopares 7 y 11 (ver Fig. 5) y resultados de la simulación de esa misma geometría y profundidades para un valor de $h=3.100 \mathrm{~W} / \mathrm{m}^{2} \mathrm{~K}$.

Figure 6. Cooling profiles obtained for the cilinder with thermocouples 7 and 11 (see Fig. 5) and simulation results for the same positions with $h=3100 \mathrm{~W} / \mathrm{m}^{2} \mathrm{~K}$.

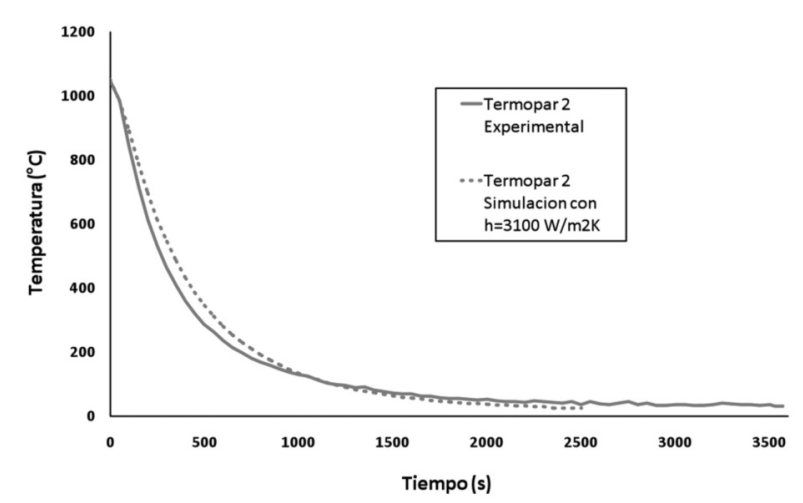

Figura 7. Perfil de enfriamiento obtenido en el conector por el termopar 2 y resultado de la simulación de esa misma geometría y profundidad para un valor de $\mathrm{h}=3.100 \mathrm{~W} / \mathrm{m}^{2} \mathrm{~K}$.

Figure 7. Cooling profile obtained for the connector by the thermocouple 2 and simulation result for the same geometry and position with $h=3100 \mathrm{~W} / \mathrm{m}^{2} \mathrm{~K}$.

ello basta con introducir la nueva geometría en ANSYS, calcular su perfil de enfriamiento en un punto interior e introducirlo en el diagrama de precipitación isoterma del material.

Para terminar con el caso estudiado se ha comparado el perfil de enfriamiento obtenido en el conec-

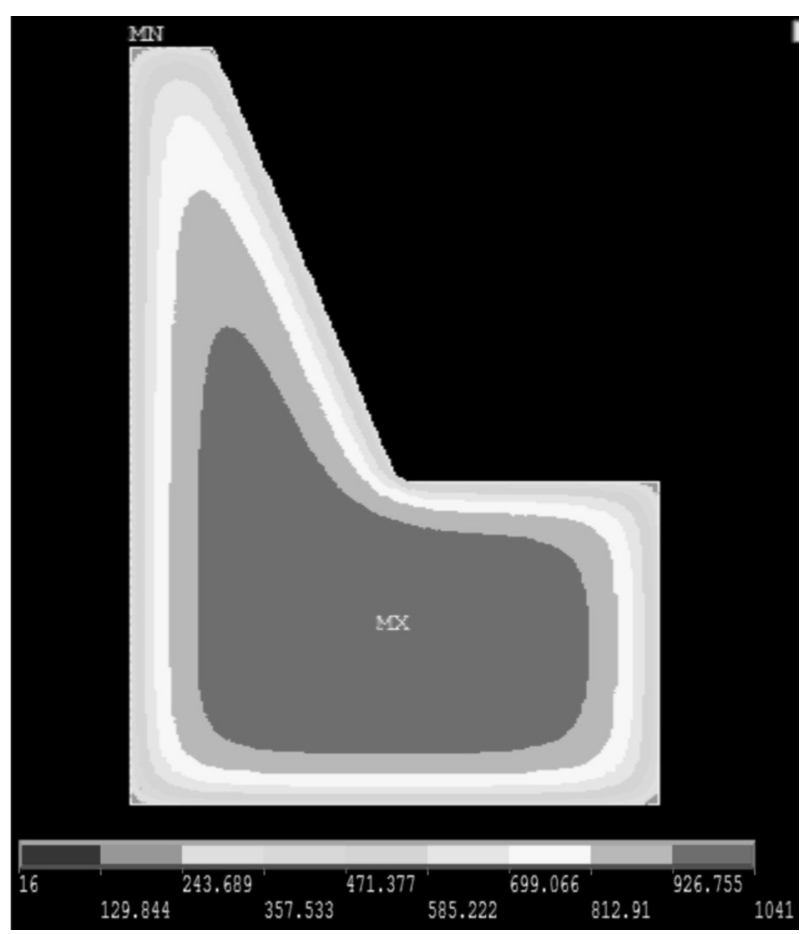

Figura 8. Temperaturas $\left({ }^{\circ} \mathrm{C}\right)$ en escala de grises en el interior del conector $50 \mathrm{~s}$ después de introducirlo en el baño.

Figure 8. Temperature $\left({ }^{\circ} \mathrm{C}\right)$ color diagram of the connector section $50 \mathrm{~s}$ after the introduction into water. 


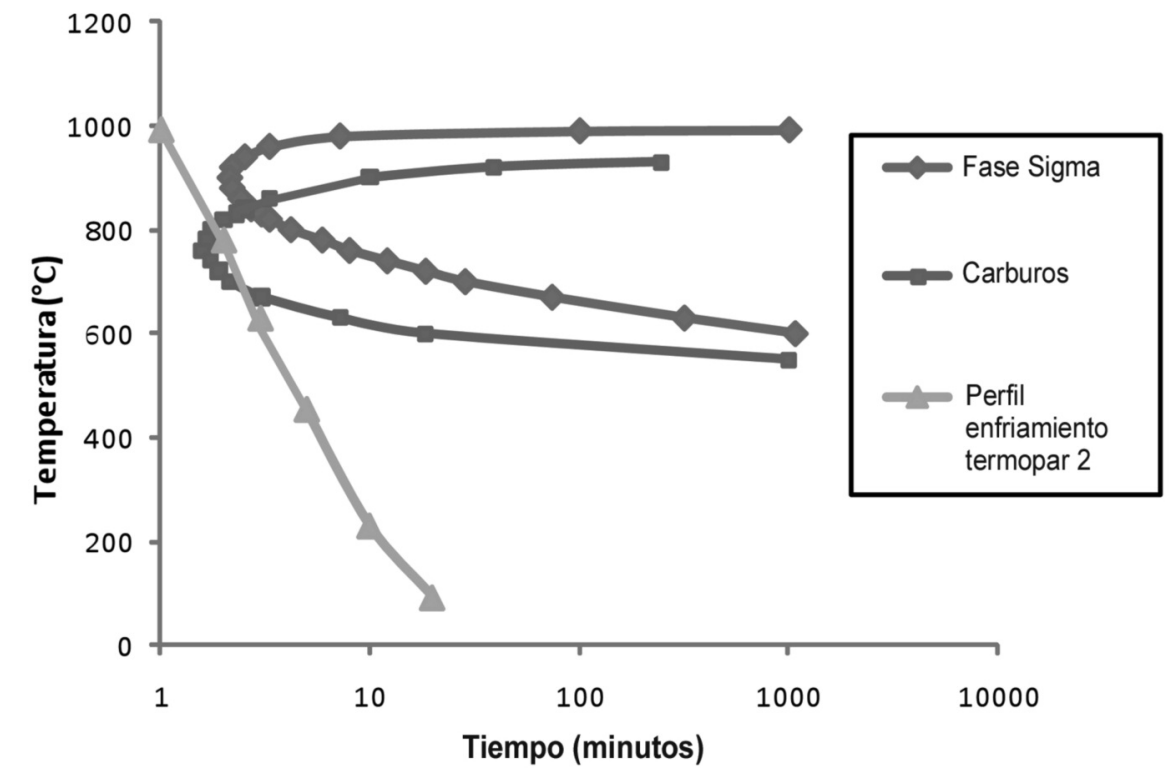

Figura 9. Perfil de enfriamiento obtenido en el conector con ANSYS y un valor de $3.100 \mathrm{~W} / \mathrm{m}^{2} \mathrm{~K}$ introducido en el diagrama de precipitación isoterma del acero SAF $2205^{[21]}$.

Figure 9. Cooling profile obtained inside the connector with ANSYS using $h=3100 \mathrm{~W} / \mathrm{m}^{2} \mathrm{~K}$ overlaid the isothermal precipitation diagram of SAF $2205^{[21] .}$

tor introduciendo los datos obtenidos con ANSYS en el diagrama de precipitación isoterma ${ }^{[21]}$ del material estudiado (Fig. 9).

Se puede observar cómo el perfil de enfriamiento sufrido por el termopar 2 del conector no cruza la curva de fase sigma aunque sí atraviesa ligeramente la de carburos.

\section{CONCLUSIONES}

- Se ha desarrollado una metodología para predecir la precipitación de fase sigma en instalaciones industriales para grandes espesores. Esta metodología nos permite calcular los perfiles de enfriamiento sin sacrificar ninguna pieza, utilizando una o varias piezas patrón sensorizadas con termopares y una sencilla simulación posterior.

- Para el caso estudiado h se estabiliza en torno a los $3.100 \mathrm{~W} / \mathrm{m}^{2} \mathrm{~K}$ mientras que en la bibliografía encontrada el valor para piezas menores se estabiliza entre $800-2.000 \mathrm{~W} / \mathrm{m}^{2} \mathrm{~K}$.

- Una vez calculado el valor de h estabilizada se pueden simular distintas geometrías de sección gruesa con muy buenos resultados a la hora de predecir perfiles de enfriamiento.
- Tal y como se suponía a partir de lo leído en la literatura, adoptando un valor de h constante se consiguen aproximar los resultados de la simulación a los resultados experimentales con mayor precisión cuanto más al interior de la pieza. Así mismo, esta precisión aumenta una vez pasados varios segundos del temple (después de la etapa de ebullición).

\section{REFERENCIAS}

[1] Det Norske Veritas, "Design of duplex stainless steel subsea equipment exposed to cathodic protection, Recommended practice Det Norske Veritas DNV-RP-F112", October 2008.

[2] T. Cassagne, Duplex 2007 International Conference \& Expo, Grado, Italia, 18-20 June, 2007.

[3] M.P. Rodríguez, J.F. Almagro, J. Botella y P. Valerga, Bol. Soc. Esp. Ceram. 43 (2004) 237 242.

[4] G. Fargas, A. Mestra, M. Anglada y A. Mateo, Anales de Mecánica de la Fractura, XXVI Encuentro del Grupo Español de Fractura, Santander, 2009, pp. 160-165. 
[5] G. Fargas, M. Anglada y A. Mateo, VIII Congreso Nacional de Propiedades Mecánicas de Sólidos, Gandía, 2002, pp. 77-86.

[6] J.L. Garin, R.L. Mannheim y M.A. Camus, Rev. Metal. 47 (2011) 296-306.

[7] C. Heming, J. Fan y H. Wang. J. Mater. Process. Tech. 63 (1997) 568-572.

[8] C.E. Bates, G.E. Totten y R.L. Brennan. Quenching of Steel, Heat Treating. Vol 4, ASM Handbook, ASM International, 1991, pp. 67-120.

[9] K. Babu y T.S. Prasanna Kumar, Metall. Mater. Trans. B 41 (2010) 214-224.

[10] B.A. Anderson y R.P. Singh. Int. J. Refrig. 74 (2006) 383-389.

[11] R. Rabindranath y P. Prakash. J. Food Eng. 110 (2012) 141-157.

[12] J. Zhang y M.A. Delichatsios, Fire Safety J. 44 (2009) 681-690.

[13] A.A. Ranjbar, M. Ezzati y M. Famouri, J. Mater. Process. Tech. 209 (2009) 5.611-5.617.
[14] H.K. Kim y S.I. Oh, J. Mater.Process. Tech. 112 (2001) 157-165.

[15] C. Heming, H. Xieqing y W. Honggang, J. Mater. Process. Tech. 89-90 (1999) 339-343.

[16] L. Huiping, Z. Guoqun, H. Lianfang y M. Yue, Measurement 41 (2008) 676-686.

[17] C. Heming, H. Xieqing y X. Jianbin, J. Mater. Process. Tech. 138 (2003) 399-402.

[18] R. Mehrotra, A. Vedhanayagam y R. Shivpuri, Transactions of NAMRI, NAMRI/SME XXI, Ontario, Canada, 1993, pp. 73-79.

[19] A.M. Osman, Ph. D. Thesis, Michigan State University, East Lansing, MI, 1987.

[20] H. Hasan, M. Peet, J. Jalil y H. Bhadeshia, Heat Mass Transfer. 47 (2011) 315-321.

[21] International Molybdenum Association (IMOA), Practical Guidelines for the Fabrication of Duplex Stainless Steels, ISBN 978-1-907470-00-4, 2009. 